\title{
On the absence of marginal pinching in thin free films
}

\author{
P. D. Howell*and H. A. Stone ${ }^{\dagger}$
}

16 August 2004

\begin{abstract}
This paper concerns the drainage of a thin liquid lamella into a Plateau border. Many models for draining soap films assume that their gas-liquid interfaces are effectively immobile. Such models predict a phenomenon known as "marginal pinching", in which the film tends to pinch in the margin between the lamella and the Plateau border. We analyse the opposite extreme, in which the gas-liquid interfaces are assumed to be stress-free. We show that no marginal pinching occurs in this case: the film always approaches a monotonic profile at large times.
\end{abstract}

\section{Introduction}

Many experimentalists have reported convective-like motions associated with soap films that have nonuniform thicknesses at their borders, a phenomenon that goes by the name of "marginal regeneration" [9]. With a view to understanding the initial stages of this process, Aradian, Raphaël and de Gennes [1] studied "marginal pinching", which refers to the development of a pinched region in the neighbourhood of the Plateau border at the edge of a soap film. Under the assumption that the fluid velocity is zero at the surfactantcovered boundaries of the film, the film thickness was found to satisfy a nonlinear partial differential equation familiar from lubrication theory (e.g. [10]). Similarity solutions were presented by treating the film in three distinct regions: a flat central film, a pinched region, and a capillary statics region at the Plateau border.

In a real soap film, there is a balance between the advection of surfactant along the interfaces, replenishment from surfactant molecules in solution, and the Marangoni stress resulting from nonuniform surface concentration of surfactant (see $[3,5]$ ). The evolution equation analysed by [1] is a limiting case, in which the free surfaces are so loaded with surfactant, and the stresses due to even small surfactant gradients so large, that the free surfaces are effectively tangentially immobile. For example, soap films made from large

\footnotetext{
*Mathematical Institute, University of Oxford, 24-29 St Giles', Oxford OX1 3LB, UK.

${ }^{\dagger}$ Division of Engineering \& Applied Sciences, Harvard University, 308 Pierce Hall, Cambridge, MA 02138, USA.
} 
(a)

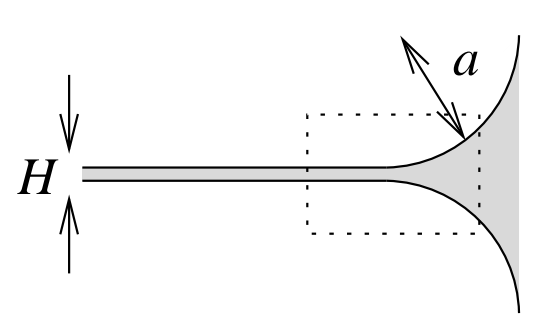

(b)

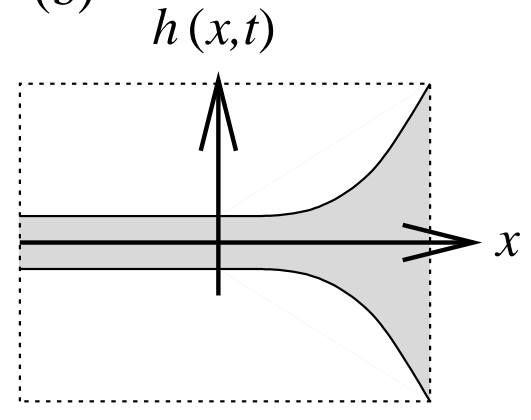

Figure 1: (a) Schematic of a thin film of thickness $H$ attached to a Plateau border of radius a. (b) Close-up of the margin between the film and the Plateau border.

molecular weight surfactants or polymers or from mixtures of surfactants such as SDS and dodecanol (e.g. [9]) are generally observed to have rigid surfaces.

Highly mobile interfaces are also possible. In these circumstances, a large bulk concentration of surfactant, generally in the form of micelles, provides a reservoir of monomer that can maintain the interface at nearly uniform surfactant concentration, thereby eliminating surfactant gradients and stresses (though the value of the surface tension can be significantly reduced). In this paper, we analyse this limit of high interfacial mobility, for which the film thickness and fluid velocity satisfy a pair of coupled nonlinear partial differential equations. Such motions of thin fluid sheets also appear in situations such as curtain coating [4, see the appendix by Taylor] and the drawing of glass sheets [8]. We find that, in contrast with the immobile case, there is no marginal pinching: the film always approaches a monotonic profile as time increases.

We set out the governing equations and boundary conditions in section 2 . In section 3 , we perform a coordinate transformation that reduces the nonlinear problem to the linear heat equation, with suitable matching conditions. A simple travelling-wave solution of the transformed problem is identified in section 4 and shown to be globally attractive in section 5. The theory is illustrated by showing the behaviour of typical time-dependent solutions in section 6 , and our results are discussed in section 7 .

\section{Problem Statement}

We consider the model problem of a semi-infinite film of uniform initial thickness $h_{0}$ attached to a Plateau border with radius of curvature $a$, as shown schematically in figure 1 . If $h^{*}$ is the film thickness and $x^{*}$ is the coordinate measuring distance along the film, then we require

$$
h^{*} \rightarrow H \quad \text { as } \quad x^{*} \rightarrow-\infty, \quad \frac{\partial^{2} h^{*}}{\partial x^{* 2}} \rightarrow \frac{2}{a} \quad \text { as } \quad x^{*} \rightarrow+\infty .
$$


These conditions imply that the following scales are appropriate in nondimensionalising $h^{*}$ and $x^{*}$ :

$$
h^{*}=H h, \quad x^{*}=\sqrt{\frac{a H}{2}} x .
$$

Thus the film has an effective aspect ratio $\sqrt{a / H}$; if $a$ is assumed to be much larger than $H$, then the dynamics may be described using a long-wave approximation.

If one assumes, as in [1], that the surfaces of the film are immobile, then the drainage timescale is found to be

$$
t^{*} \sim t_{\mathrm{i}}=\frac{3 \mu a^{2}}{4 \gamma H}
$$

where $\mu$ and $\gamma$ are, respectively, the viscosity and surface tension of the liquid comprising the film. In this case, the evolution of the film thickness is governed by the dimensionless lubrication equation

$$
\frac{\partial h}{\partial t}+\frac{\partial}{\partial x}\left(h^{3} \frac{\partial^{3} h}{\partial x^{3}}\right)=0
$$

If, however, the liquid-air interfaces are stress-free, then drainage occurs over the timescale

$$
t^{*} \sim t_{\mathrm{s- \textrm {f }}}=\frac{4 \mu a}{\gamma}
$$

which is a factor of $H / a$ smaller than the immobile timescale $t_{\mathrm{i}}$. In this case, the film thickness $h$ and tangential liquid velocity $u$ satisfy the coupled dimensionless equations $[6,8]$

$$
\begin{aligned}
\frac{\partial h}{\partial t}+\frac{\partial}{\partial x}(u h) & =0 \\
\frac{\partial}{\partial x}\left(h \frac{\partial u}{\partial x}\right)+h \frac{\partial^{3} h}{\partial x^{3}} & =0
\end{aligned}
$$

where time has been scaled by $t_{\mathrm{s}-\mathrm{f}}$ and velocity by $\sqrt{a H / 2} / t_{\mathrm{s}-\mathrm{f}}$. The system (6) is to be solved subject to the boundary conditions

$$
h \rightarrow 1 \quad \text { as } \quad x \rightarrow-\infty, \quad \frac{\partial^{2} h}{\partial x^{2}} \rightarrow 1 \quad \text { as } \quad x \rightarrow+\infty
$$

and the initial condition

$$
h=h_{0}(x) \text { at } t=0 .
$$

Generic solutions of the so-called lubrication equation (4), subject to the boundary conditions (7), were shown by [1] to exhibit "marginal pinching". At large times, the film thickness approaches a non-monotonic profile, attaining its continually decreasing minimum value in the margin between the lamella and the Plateau border. In this paper we examine whether or not solutions of the system (6) exhibit qualitatively similar behaviour. 


\section{Problem transformation}

Analysis of (6b) as $x$ approaches $-\infty$ reveals that $u$ must approach a function only of $t$. Since the system (6) is invariant under a time-dependent translation in $x$, we may choose the coordinate axes such that

$$
u \rightarrow 0 \quad \text { as } \quad x \rightarrow-\infty
$$

Then (6b) may be integrated once with respect to $x$, applying the conditions as $x \rightarrow-\infty$ to obtain

$$
h \frac{\partial u}{\partial x}=\frac{1}{2}\left(\frac{\partial h}{\partial x}\right)^{2}-h \frac{\partial^{2} h}{\partial x^{2}},
$$

which may be rewritten as

$$
\frac{1}{\sqrt{h}} \frac{\partial u}{\partial x}=-\frac{\partial}{\partial x}\left(\frac{1}{\sqrt{h}} \frac{\partial h}{\partial x}\right)=-2 \frac{\partial^{2}}{\partial x^{2}}(\sqrt{h}) .
$$

Now we transform variables from $(x, t)$ to $(\xi, t)$, where $\xi$ is defined by

$$
\xi=x+\int_{-\infty}^{x}\left(\frac{1}{\sqrt{h\left(x^{\prime}, t\right)}}-1\right) \mathrm{d} x^{\prime}
$$

so that

$$
\frac{\partial \xi}{\partial x}=\frac{1}{\sqrt{h}} .
$$

Using (6a), we calculate $\partial \xi / \partial t$ as follows:

$$
\begin{aligned}
\frac{\partial \xi}{\partial t} & =-\frac{1}{2} \int_{-\infty}^{x} \frac{1}{h\left(x^{\prime}, t\right)^{3 / 2}} \frac{\partial h\left(x^{\prime}, t\right)}{\partial t} \mathrm{~d} x^{\prime}=\frac{1}{2} \int_{-\infty}^{x} \frac{1}{h\left(x^{\prime}, t\right)^{3 / 2}} \frac{\partial}{\partial x^{\prime}}\left(u\left(x^{\prime}, t\right) h\left(x^{\prime}, t\right)\right) \mathrm{d} x^{\prime} \\
& =\frac{1}{2} \int_{-\infty}^{x}\left(\frac{1}{\sqrt{h\left(x^{\prime}, t\right)}} \frac{\partial u\left(x^{\prime}, t\right)}{\partial x^{\prime}}+\frac{u\left(x^{\prime}, t\right)}{h\left(x^{\prime}, t\right)^{3 / 2}} \frac{\partial h\left(x^{\prime}, t\right)}{\partial x^{\prime}}\right) \mathrm{d} x^{\prime} \\
& =-\frac{u(x, t)}{\sqrt{h(x, t)}}+\frac{3}{2} \int_{-\infty}^{x} \frac{1}{\sqrt{h\left(x^{\prime}, t\right)}} \frac{\partial u\left(x^{\prime}, t\right)}{\partial x^{\prime}} \mathrm{d} x^{\prime}
\end{aligned}
$$

after integrating the second term by parts. Now we use the expression (11) to obtain

$$
\frac{\partial \xi}{\partial t}=-\frac{1}{\sqrt{h}}\left(u+\frac{3}{2} \frac{\partial h}{\partial x}\right)
$$

Thus, the chain rules to transform to the new coordinates are

$$
\frac{\partial}{\partial x} \mapsto \frac{1}{\sqrt{h}} \frac{\partial}{\partial \xi}, \quad \frac{\partial}{\partial t} \mapsto \frac{\partial}{\partial t}-\frac{1}{\sqrt{h}}\left(u+\frac{3}{2} \frac{\partial h}{\partial x}\right) \frac{\partial}{\partial \xi}
$$


As a consequence, equation (6a) is transformed to

$$
\frac{\partial h}{\partial t}=\frac{\partial^{2} h}{\partial \xi^{2}}+\frac{1}{2 h}\left(\frac{\partial h}{\partial \xi}\right)^{2},
$$

or

$$
\sqrt{h} \frac{\partial h}{\partial t}=\frac{\partial}{\partial \xi}\left(\sqrt{h} \frac{\partial h}{\partial \xi}\right)
$$

Thus, the function $\phi=h^{3 / 2}$ satisfies the linear heat equation

$$
\frac{\partial \phi}{\partial t}=\frac{\partial^{2} \phi}{\partial \xi^{2}}
$$

To obtain the boundary conditions for $\phi$ we must examine the behaviour as $x \rightarrow \infty$, that is as the film approaches the Plateau border. If $h$ has a Laurent expansion

$$
h \sim \frac{x^{2}}{2}+B(t) x+C(t)+\frac{a_{1}(t)}{x}+\frac{a_{2}(t)}{x^{2}}+\cdots \quad \text { as } \quad x \rightarrow \infty,
$$

where $B, C, a_{1}, a_{2}, \ldots$ are all arbitrary functions of $t$, then we have

$$
\begin{aligned}
\frac{\partial \xi}{\partial x}= & \frac{1}{\sqrt{h}} \sim \frac{\sqrt{2}}{x}-\frac{\sqrt{2} B(t)}{x^{2}}+\cdots \\
& \Rightarrow \xi \sim \sqrt{2} \log x+D(t)+\frac{\sqrt{2} B(t)}{x}+\cdots \\
& \Rightarrow x \sim \exp \left(\frac{\xi-D(t)}{\sqrt{2}}\right) \\
& \Rightarrow \phi \sim \frac{1}{2 \sqrt{2}} \exp \left(\frac{3}{\sqrt{2}}(\xi-D(t))\right) \quad \text { as } \quad \xi \rightarrow \infty
\end{aligned}
$$

where $D$ is a further arbitrary function of $t$. Substitution of (24) into (19) leads to

$$
-\frac{3}{\sqrt{2}} \frac{\mathrm{d} D}{\mathrm{~d} t}=\frac{9}{2} \Rightarrow D=D_{0}-\frac{3}{\sqrt{2}} t
$$

where $D_{0}$ is determined by the initial conditions. Specifically, the definition (22) may be used to express $D_{0}$ in the form

$$
D_{0}=1+\int_{-\infty}^{1}\left(\frac{1}{\sqrt{h_{0}}}-1\right) \mathrm{d} x+\int_{1}^{\infty}\left(\frac{1}{\sqrt{h_{0}}}-\frac{\sqrt{2}}{x}\right) \mathrm{d} x .
$$

Thus the asymptotic behaviour of $\phi$ is

$$
\begin{array}{rlrl}
\phi & \rightarrow 1 & \text { as } \xi \rightarrow-\infty, \\
\phi & \sim \frac{1}{2 \sqrt{2}} \exp \left(\frac{3}{\sqrt{2}}\left(\xi-D_{0}\right)+\frac{9}{2} t\right) \quad \text { as } \xi \rightarrow+\infty
\end{array}
$$




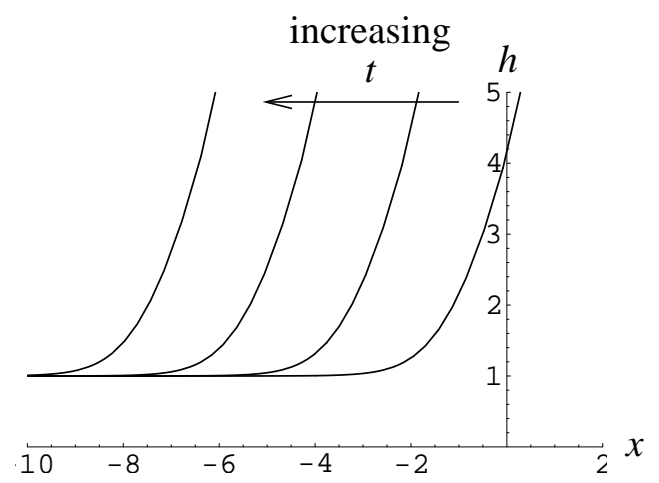

Figure 2: Film thickness $h$ versus distance $x$, as given by (30) and (32), for values of $t=1,2,3,4$. This is the travelling-wave solution, propagating right to left.

along with the obvious initial condition

$$
\phi=\phi_{0}(\xi) \quad \text { at } \quad t=0, \quad \phi_{0}(\xi)=\left[h_{0}(x(\xi, 0))\right]^{3 / 2} .
$$

We have therefore reduced the original nonlinear coupled partial differential equations (6) to the heat equation (19), subject to the boundary and initial conditions (27)-(28).

\section{Travelling-wave solution}

Now, we can identify an exact solution of (19) that also satisfies the matching conditions (27), namely

$$
\phi_{T}(\xi, t)=1+\frac{1}{2 \sqrt{2}} \exp \left(\frac{3}{\sqrt{2}}\left(\xi-D_{0}\right)+\frac{9}{2} t\right) .
$$

The film thickness profile corresponding to (29) is recovered via

$$
\begin{aligned}
h(x, t)= & \phi_{T}(\xi, t)^{2 / 3}, \text { where } \\
x= & \xi+\int_{-\infty}^{\xi}\left(\phi_{T}\left(\xi^{\prime}, t\right)^{1 / 3}-1\right) \mathrm{d} \xi^{\prime} \\
\Rightarrow x= & D_{0}-\frac{3}{\sqrt{2}} t-\left(\frac{18+\sqrt{3} \pi-9 \log 6}{9 \sqrt{2}}\right)+\left(2 \sqrt{2}+\mathrm{e}^{3\left(\xi-D_{0}\right) / \sqrt{2}+9 t / 4}\right)^{1 / 3} \\
& -2 \mathrm{e}^{-\sqrt{2}\left(\xi-D_{0}\right)-3 t} \mathrm{~F}\left(\frac{2}{3} ; \frac{2}{3} ; \frac{5}{3} ;-2 \sqrt{2} \mathrm{e}^{-3\left(\xi-D_{0}\right) / \sqrt{2}-9 t / 4}\right)
\end{aligned}
$$

and $\mathrm{F}$ is a hypergeometric function [7, page 1065]. This solution is plotted in figure 2 .

Now, (29) and (30) imply that $h$ is a function only of the group $(\xi+3 t / \sqrt{2})$ which itself, by (32), is a function only of $x+3 t / \sqrt{2}$. Thus (29) corresponds to a travelling-wave solution of the original system (6), as suggested by figure 2. With this insight, we can obtain the solution more directly as follows. 
Suppose that $h$ and $u$ are functions only of $\eta=x-c t$, where the constant wavespeed $c$ is to be determined. Then (6a) and the matching conditions (7) as $x \rightarrow-\infty$ imply that

$$
(u-c) h=-c \Rightarrow \frac{\mathrm{d} u}{\mathrm{~d} \eta}=\frac{c}{h^{2}} \frac{\mathrm{d} h}{\mathrm{~d} \eta} .
$$

Now we substitute $\mathrm{d} u / \mathrm{d} \eta$ from (33) into (11) to obtain

$$
\frac{c}{h^{5 / 2}} \frac{\mathrm{d} h}{\mathrm{~d} \eta}=-\frac{\mathrm{d}}{\mathrm{d} \eta}\left(\frac{1}{\sqrt{h}} \frac{\mathrm{d} h}{\mathrm{~d} \eta}\right) \Rightarrow \frac{\mathrm{d} h}{\mathrm{~d} \eta}=\frac{2 c}{3}\left(\frac{1}{h}-\sqrt{h}\right)
$$

again using the matching conditions (7) at $-\infty$. Now the behaviour $h \sim \eta^{2} / 2$ as $\eta \rightarrow \infty$ gives

$$
x \sim \frac{2 c}{3}\left(-\frac{x}{\sqrt{2}}\right) \Rightarrow c=-\frac{3}{\sqrt{2}} .
$$

This result agrees with the propagation speed deduced from (30) and (32). Then, one further integration of (34) leads to the solution in the form

$$
2 \sqrt{h}-\frac{2}{\sqrt{3}} \tan ^{-1}\left(\frac{1+2 \sqrt{h}}{3}\right)+\frac{1}{3} \log \left(\frac{h-2 \sqrt{h}+1}{h+\sqrt{h}+1}\right)=\sqrt{2}\left(\eta-\eta_{0}\right),
$$

where $\eta=x-c t$ and $\eta_{0}$ is the arbitrary initial translation. This travelling-wave solution is equivalent to the steady solution of (6) obtained by [3], subject to a steady translation. Plots of (36) agree exactly with figure 2. Notice that, in this solution, $h$ is a monotonic increasing function of $\eta$, so that there is no "marginal pinch".

\section{Global stability}

Clearly, the simple travelling wave (36) is not the only solution of (6) satisfying boundary conditions (7): the general solution must depend also on the initial film profile $h_{0}(x)$. We will now show, however, that (36) is globally attractive, so that any initial condition will ultimately take this form. To establish this property, we first examine more carefully the matching conditions as $x \rightarrow+\infty$ and the film approaches the constant-curvature Plateau border. With $h$ given asymptotically by (20), (6b) gives the corresponding form of $u$ as

$$
u \sim A(t)-\frac{B(t)^{2}-2 C(t)}{x}+\frac{3 a_{1}(t)+B(t)^{3}-2 B(t) C(t)}{x^{2}}+\cdots \quad \text { as } \quad x \rightarrow \infty,
$$


where $A$ is a further arbitrary function of $t$. Ordinary differential equations relating these coefficients are obtained by substituting (20) and (37) into (6a):

$$
\begin{aligned}
\frac{\mathrm{d} B}{\mathrm{~d} t} & =-A \\
\frac{\mathrm{d} C}{\mathrm{~d} t} & =-C-A B+\frac{1}{2} B^{2} \\
\frac{\mathrm{d} a_{1}}{\mathrm{~d} t} & =0 \\
\frac{\mathrm{d} a_{2}}{\mathrm{~d} t} & =2 a_{2}+(A+2 B) a_{1}+\frac{\left(B^{2}-2 C\right)^{2}}{3},
\end{aligned}
$$

and so forth.

By substituting (20) into (12), we obtain corrections to the expansions (22) and (24) as follows

$$
\begin{aligned}
\xi & \sim \sqrt{2} \log x+D+\frac{\sqrt{2} B}{x}+\frac{2 C-3 B^{2}}{2 \sqrt{2} x^{2}}+\frac{2 a_{1}+5 B^{3}-6 B C}{3 \sqrt{2} x^{3}}+\cdots \\
\Rightarrow x & \sim \mathrm{e}^{(\xi-D) / \sqrt{2}}-B+\frac{B^{2}-2 C}{4} \mathrm{e}^{-(\xi-D) / \sqrt{2}}-\frac{a_{1}}{3} \mathrm{e}^{-\sqrt{2}(\xi-D)}+\cdots \\
\Rightarrow \phi & \sim \frac{1}{2 \sqrt{2}} \mathrm{e}^{3(\xi-D) / \sqrt{2}}-\frac{3\left(B^{2}-2 C\right)}{8 \sqrt{2}} \mathrm{e}^{(\xi-D) / \sqrt{2}}+\frac{a_{1}}{2}+O\left(\mathrm{e}^{-(\xi-D) / \sqrt{2}}\right)
\end{aligned}
$$

as $\xi \rightarrow \infty$. Now, (38c) implies that $a_{1}$ is constant in time and, by combining (38a) and (38b), we find that the translation-invariant combination $B^{2}-2 C$ satisfies

$$
\begin{aligned}
& \frac{\mathrm{d}}{\mathrm{d} t}\left(B^{2}-2 C\right)=-\left(B^{2}-2 C\right) \\
& \Rightarrow\left(B^{2}-2 C\right)=\left(B_{0}^{2}-2 C_{0}\right) \mathrm{e}^{-t} .
\end{aligned}
$$

Thus the asymptotic behaviour of $\phi$ is

$$
\phi \sim \frac{1}{2 \sqrt{2}} \mathrm{e}^{3\left(\xi-D_{0}\right) / \sqrt{2}+9 t / 2}-\frac{3\left(B_{0}^{2}-2 C_{0}\right)}{8 \sqrt{2}} \mathrm{e}^{\left(\xi-D_{0}\right) / \sqrt{2}+t / 2}+\frac{a_{1}}{2}+\cdots
$$

as $\xi \rightarrow \infty$. All the coefficients in (44) may be determined in principle from the initial film profile $h_{0}(x)$, since $\phi(\xi, 0)$ is related to $h_{0}(x)$ by equation $(28)$.

A solution of the heat equation that satisfies (44) and also approaches 1 as $\xi \rightarrow-\infty$ is

$$
\begin{aligned}
\Phi= & \frac{1}{2 \sqrt{2}} \mathrm{e}^{3\left(\xi-D_{0}\right) / \sqrt{2}+9 t / 2}-\frac{3\left(B_{0}^{2}-2 C_{0}\right)}{8 \sqrt{2}} \mathrm{e}^{\left(\xi-D_{0}\right) / \sqrt{2}+t / 2} \\
& +\frac{1}{2}\left(\frac{a_{1}}{\sqrt{2}}+1\right)+\frac{1}{2}\left(\frac{a_{1}}{\sqrt{2}}-1\right) \operatorname{erf}\left(\frac{\xi}{2 \sqrt{t}}\right)
\end{aligned}
$$


Thus, if we set $\phi=\Phi+\tilde{\phi}$, then $\tilde{\phi}$ satisfies

$$
\begin{aligned}
\frac{\partial \tilde{\phi}}{\partial t} & =\frac{\partial^{2} \tilde{\phi}}{\partial \xi^{2}} \\
\tilde{\phi} & \rightarrow 0 \\
\tilde{\phi} & =\phi_{0}(\xi)-\Phi(\xi, 0)
\end{aligned}
$$

which implies that $\tilde{\phi} \rightarrow 0$ as $t \rightarrow \infty$. Thus the solution $\Phi$ is globally attractive and, hence, the large-time behaviour of $h$ is given by

$$
\begin{aligned}
h(x, t) & =\Phi(\xi, t)^{2 / 3} \\
x & =\xi+\int_{-\infty}^{\xi}\left(\Phi\left(\xi^{\prime}, t\right)^{1 / 3}-1\right) \mathrm{d} \xi^{\prime} .
\end{aligned}
$$

Notice that $\Phi$ reduces to the travelling-wave solution $\phi_{T}$ (equation 29) if $B_{0}^{2}-2 C_{0}=0$ and $a_{1}=\sqrt{2}$.

As $t \rightarrow \infty$, the first term in (45) dominates the second except where $\xi-D_{0} \lesssim-2 \sqrt{2} t$, in which case both are smaller than $\mathrm{e}^{-3 t / 2}$. The dominant behaviour of $\Phi$ at large $t$ is, therefore,

$$
\begin{array}{llrl}
\Phi & \sim \frac{1}{2 \sqrt{2}} \mathrm{e}^{3\left(\xi-D_{0}\right) / \sqrt{2}+9 t / 2} & \xi-D_{0} \gg-\frac{3 t}{\sqrt{2}}, \\
\Phi & \sim 1 & \xi-D_{0} \ll-\frac{3 t}{\sqrt{2}}, \\
\Phi & \sim 1+\frac{1}{2 \sqrt{2}} \mathrm{e}^{3\left(\xi-D_{0}\right) / \sqrt{2}+9 t / 2} & \xi-D_{0} \sim-\frac{3 t}{\sqrt{2}} .
\end{array}
$$

In other words, the travelling-wave solution $\phi_{T}$ is always the dominant behaviour as $t \rightarrow \infty$; the extra exponential and error-function terms in (45) just provide decaying transient effects.

\section{Sample solutions}

We illustrate the global stability behaviour described above in figure 3. First, figure 3(a) shows the evolution of the film thickness when $B_{0}^{2}-2 C_{0}=10$ and $a_{1}=\sqrt{2}$. This initial shape corresponds to an increased slope in the parabola at plus infinity and causes a dip in the profile close to the meniscus. As time increases, though, the amplitude of the pinch decays rapidly and the travelling-wave solution (propagating from right to left) is recovered. Second, in figure $3(\mathrm{~b})$ we set $B_{0}^{2}-2 C_{0}=-10$ and $a_{1}=\sqrt{2}$, corresponding to a decreased initial slope at plus infinity. The initial profile is noticeably flatter but, again, the travelling-wave solution soon emerges.

In figure 4 , we illustrate solutions with $B_{0}^{2}-2 C_{0}=0$ and different values of $a_{1}$. Recall that $a_{1}$ is the coefficient of $1 / x$ in the large- $x$ expansion of $h$. Not surprisingly, it has a 

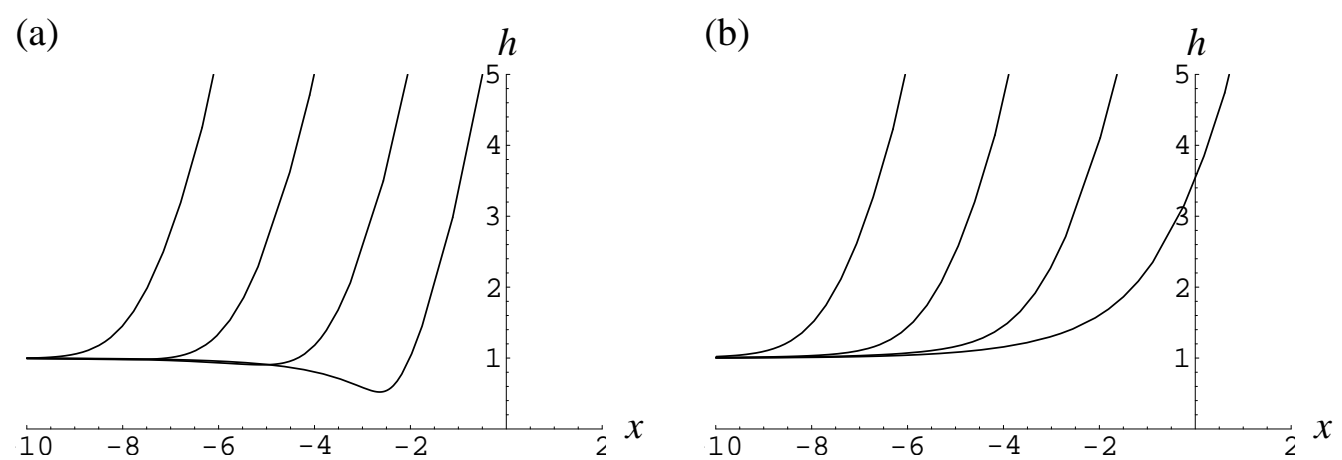

Figure 3: Film thickness $h$ versus distance $x$ for values of $t=1,2,3,4$. Parameter values (a) $B_{0}^{2}-2 C_{0}=10, a_{1}=\sqrt{2}, D_{0}=0$; (b) $B_{0}^{2}-2 C_{0}=-10, a_{1}=\sqrt{2}, D_{0}=0$.

(a)

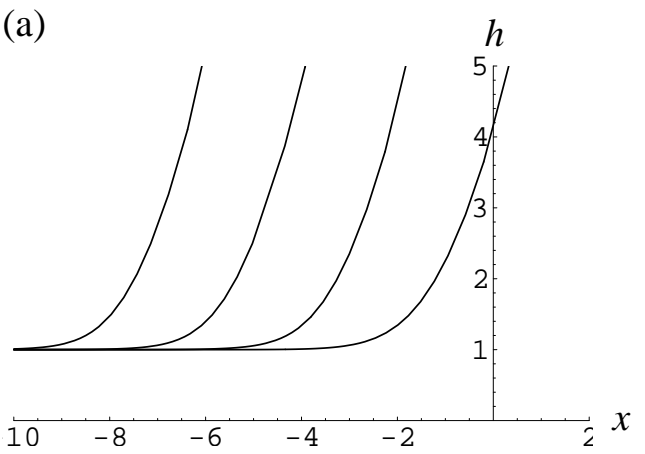

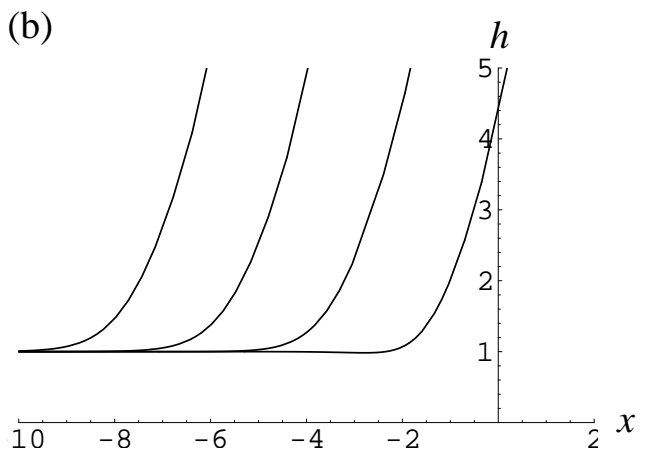

Figure 4: Film thickness $h$ versus distance $x$ for values of $t=1,2,3,4$. Parameter values (a) $B_{0}^{2}-2 C_{0}=0, a_{1}=5, D_{0}=0$; (b) $B_{0}^{2}-2 C_{0}=0, a_{1}=-5, D_{0}=0$. 
somewhat smaller influence on the qualitative behaviour than does $B_{0}^{2}-2 C_{0}$. In figure $4(\mathrm{a})$, $a_{1}=5$, which leads to a slight decrease in the slope at plus infinity and thus a flatter profile. In figure $4(\mathrm{~b}), a_{1}$ is set to -5 , which increases the slope at plus infinity and gives rise to a small pinch near the meniscus. In both cases, the effects decay as $t$ increases and the travelling-wave solution again emerges as the dominant behaviour.

\section{Discussion}

We have shown that a stress-free thin liquid film, draining into a Plateau border, tends to a monotonic shape, irrespective of its initial profile. In contrast, a film whose liquid-air interfaces are immobile approaches a non-monotonic profile, with a pinch in the margin between the film and the Plateau border. Either scenario is a limiting case of a true lamella, in which various physical effects compete to render the surfaces partially mobile. For example, Breward \& Howell [3] consider a lamella stabilised by soluble surfactant and find that both monotonic and nonmonotonic profiles are possible. Similarly, Braun et al. [2] model the gravity-driven drainage of a lamella into a bath, including insoluble surfactant and surface viscosity, and find that the film may or may not be monotonic, depending on the rigidity of the interfaces. None of these authors, though, was able to give a theoretical criterion to distinguish solutions that do or do not exhibit marginal pinching. We plan to analyse this transition in the future.

We found that the globally attractive solution of (6) is a travelling wave, in which the Plateau border (with radius of curvature $a$ ) propagates towards the film (of thickness $H$ ) at dimensional speed

$$
c^{*}=\frac{3 \gamma}{8 \mu} \sqrt{\frac{a}{H}},
$$

where $\gamma$ and $\mu$ are the surface tension and viscosity respectively. This counterintuitive result is partly a consequence of our choosing a reference frame in which the film velocity far from the Plateau border is zero. In a typical experiment, in which the Plateau border is fixed, the film would flow into the Plateau border at speed $c^{*}$. Furthermore, we have analysed the model problem of a semi-infinite film, whose thickness approaches a constant value $H$ at infinity. The case of a film whose length is finite, although significantly longer than $\sqrt{a H}$, may be analysed using similar methods, with the film thickness $H$ varying slowly with time as the film drains (see [3]).

This drainage is symptomatic of a qualitative difference in behaviour between our model equations (6) and the lubrication equation (4). The high-curvature free surfaces cause a large negative capillary pressure in the Plateau border, which tends to suck fluid in from the thin film. If the surfaces are stress-free, this suction is transmitted throughout the film, via the two real characteristics $t=$ const of (6). (These characteristics correspond to the two first integrals of (6) obtained in section 3.) If the free surfaces are immobile, however, the influence of the Plateau border on the film is confined to the margin between them, and this is reflected in the parabolic character of (4). 
Our analysis hinges on a coordinate transformation that converts the nonlinear coupled equations (6) for the film thickness $h$ and velocity $u$ into the linear heat equation. This transformation may well prove useful in other applications of these model equations.

\section{Acknowledgements}

We thank A. Aradian, E. Raphaël and P.-G. de Gennes for useful conversations. HAS thanks the Harvard MRSEC for partial support.

\section{References}

[1] A. Aradian, E. Raphä̈l \& P.-G. De Gennes, 2001 "Marginal pinching" in soap films. Europhys. Lett. 55(6), 834-840.

[2] R. J. Braun, S. A. Snow \& S. Naire, 2002 Models for gravitationally-driven free-film drainage. J. Engng Math. 43, 281-314.

[3] C. J. W. Breward \& P. D. Howell, 2002 The drainage of a foam lamella. J. Fluid Mech. 458, 379-406.

[4] D. R. Brown, 1961 A study of the behaviour of a thin sheet of moving liquid. J. Fluid Mech. 10, 297-305.

[5] J.-M. Chomaz, 2001 The dynamics of a viscous soap film with soluble surfactant. J. Fluid Mech. 442, 387-409.

[6] T. Erneux \& S. H. Davis, 1993 Nonlinear rupture of free films. Phys. Fluids A 5, $1117-1122$.

[7] I. S. Gradshteyn \& I. M. Ryzhik. 1994 Table of Integrals, Series, and Products. Fifth Edition. Academic Press.

[8] P. D. Howell, 1996 Models for thin viscous sheets. Euro. J. Appl. Math. 7, 321-343.

[9] K. J. Mysels, K. Shinoda \& S. Frankel, 1959 Soap Films, Studies of Their Thinning and a Bibliography (Pergamon Press, New York).

[10] A. Oron, S. H. Davis \& S. G. Bankoff, 1997 Long-scale evolution of thin liquid films. Rev. Modern Phys. 69, 931-980. 